\title{
Suppression of Toll-like receptor-mediated innate immune responses at the ocular surface by the membrane-associated mucins MUC1 and MUC16
}

\author{
BB Menon ${ }^{1}$, C Kaiser-Markoํ, S Spurr-Michaud ${ }^{1}$, AS Tisdale ${ }^{1}$ and IK Gipson ${ }^{1}$
}

Membrane-associated mucins (MAMs) expressed on the ocular surface epithelium form a dense glycocalyx that is hypothesized to protect the cornea and conjunctiva from external insult. In this study, the hypothesis that the MAMs MUC1 and MUC16, expressed on the apical surface of the corneal epithelium, suppress Toll-like receptor (TLR)-mediated innate immune responses was tested. Using an in vitro model of corneal epithelial cells that are cultured to express MAMs, we show that reduced expression of either MUC1 or MUC16 correlates with increased message and secreted protein levels of the proinflammatory cytokines interleukin (IL)-6, IL-8, and tumor necrosis factor- $\alpha$ (TNF- $\alpha$ ) following exposure of cells to the TLR2 and TLR5 agonists, heat-killed Listeria monocytogenes and flagellin, respectively. As mice express Muc1 (but not Muc16) in the corneal epithelium, a Muc1 ${ }^{-1-}$ mouse model was used to extend in vitro findings. Indeed, IL-6 and TNF- $\alpha$ message levels were increased in the corneal epithelium of Muc1 ${ }^{-1-}$ mice, in comparison with wild-type mice, following exposure of enucleated eyes to the TLR2 and TLR5 agonists. Our results suggest that the MAMs MUC1 and MUC16 contribute to the maintenance of immune homeostasis at the ocular surface by limiting TLR-mediated innate immune responses.

\section{INTRODUCTION}

The ocular surface epithelium comes in frequent contact with a variety of microorganisms, some of which can be pathogenic. However, multiple layers of protection, which include components of the mucosal barrier, ensure that the ocular surface epithelium is protected from pathogens.

Intrinsic to the mucosal barrier of all wet-surfaced epithelia of the body are a family of heavily $O$-glycosylated proteins known as mucins, existing in secreted and membraneassociated forms. Secreted mucins are synthesized by goblet cells and contain cysteine-rich domains at their $\mathrm{N}$ - and $\mathrm{C}$-termini to facilitate multimerization. ${ }^{1}$ Membrane-associated mucins (MAMs) possess a hydrophobic transmembrane domain by which they are anchored to the apical cell membrane. ${ }^{2}$ Secreted mucins, which form the bulk of mucous, remain in constant motion over epithelial surfaces and trap debris, whereas MAMs form a continuous glycocalyx on the apical side of epithelia and constitute a protective barrier. To date, nearly 10 different MAMs have been identified. These include MUCs 1, 3A/3B, 4, 12, 13, 15, 16, 17, 20, and 21 (mucins in humans and mice are abbreviated as MUC and Muc, respectively). ${ }^{3,4}$ The cytoplasmic tail (CT) of MUC1 is known to participate in signal transduction cascades. ${ }^{3,5}$ Whereas MUC1 is ubiquitously expressed across all mucosal epithelia, all other MAMs exhibit variable expression and distribution patterns. The MAM repertoire of the ocular surface epithelium consists primarily of MUCs 1,4 , and $16 .{ }^{6}$ On the ocular surface, MUCs 1 and 16 are expressed on the corneal and conjunctival epithelia, whereas MUC4 is expressed on the conjunctival epithelium. ${ }^{6,7}$ Multiple lines of investigation have suggested that MUC16 is the major contributor of barrier function on the ocular surface. ${ }^{8,9}$ Recent work using an in vitro corneal epithelial cell culture system indicates that MUC16, but not MUC1, contributes to epithelial barrier function as assessed by dye penetrence and bacterial adhesion/invasion assays and by measurements of transepithelial resistance. ${ }^{9}$ As of yet, biological functions for MUC1 on the ocular surface epithelium have not been defined.

The innate immune system of the ocular surface, principal components of which include Toll-like receptors (TLRs), also 
plays a crucial role in the early response against pathogens. To date, 10 functional TLRs have been identified in humans and 12 in mice. ${ }^{10}$ These receptors sense pathogen-associated molecular patterns intrinsic to microorganisms. Ligation of pathogen-associated molecular patterns to TLRs triggers signaling pathways that activate the transcription factors nuclear factor- $\kappa \mathrm{B}(\mathrm{NF}-\mathrm{\kappa B})$ and interferon regulatory factor-3. These factors then induce the expression of proinflammatory cytokines and antimicrobial peptides to neutralize invading pathogens. Several studies have documented the role of TLRs at the ocular surface in protection against pathogens. ${ }^{11-17}$

Although TLR-mediated immune responses against pathogens are protective, an uncontrolled response can lead to the unnecessary production of proinflammatory cytokines and cause bystander tissue damage. ${ }^{18,19}$ Thus, tight control of such responses is as crucial as clearing of invading pathogens. Recently, MUC1 and MUC13 have been shown to modulate TLR-mediated inflammatory processes in the airway and gastric epithelia. ${ }^{20-23}$ The earliest evidence suggesting an anti-inflammatory role for MUC1 came from experiments involving lung infection of $\mathrm{Mucl}^{-1-}$ mice by Pseudomonas aeruginosa. ${ }^{24}$ $\mathrm{Mucl}^{-1-}$ mice showed decreased lung colonization by $P$. aeruginosa, increased leukocytic recruitment, and elevated keratinocyte chemoattractant and tumor necrosis factor- $\alpha$ (TNF- $\alpha$ ) levels in the bronchoalveolar lavage fluid when compared with their wild-type (WT) littermates. ${ }^{24}$ Further studies showed that (i) MUC1 suppresses NF- $\mathrm{KB}$ activation in response to TLR2, 3, 4, 7, and 9 agonists, thereby suggesting that MUC1 may be a universal regulator of TLR signaling;, ${ }^{20}$ (ii) MUC1 deletion promotes dendritic cell response to TLR4 and TLR5 signaling pathways; ${ }^{25}$ and (iii) the CT of MUC1 regulates TLR3 and TLR5 signaling by associating with these TLRs and inhibiting recruitment of TRIF (TIR-domain-containing adapter-inducing interferon- $\beta$ ) and MyD88 (myeloid differentiation factor 88 ) to these receptors. ${ }^{26,27}$ Intriguingly, unlike MUC1, MUC13 was found to be proinflammatory in the intestinal epithelium. ${ }^{21}$ Given these opposing immunomodulatory roles and considering that different wet-surfaced epithelia of the body express a variable repertoire of MAMs, it is important to understand how specific MAMs regulate inflammatory processes across epithelial surfaces. With regard to the ocular surface, no information is available about the potential of MAMs to regulate inflammatory responses. Moreover, an immunomodulatory function for MUC16 has not been reported. Here, we investigate the roles of MUC1 and MUC16 in modulating TLR-mediated innate immune responses at the corneal epithelium and report that each MAM suppresses TLR-induced expression of the proinflammatory cytokines IL-6, IL-8, and TNF- $\alpha$.

\section{RESULTS}

Differentiated corneal epithelial cells express TLRs 2, 5, 6, and 10

Several laboratories have reported the expression of multiple TLRs by the ocular surface that respond to a broad range of pathogen-associated molecular patterns. ${ }^{11,14,28,29}$ Studies have also reported contrasting views about the expression, distribution, and function of TLRs in the corneal epithelium. ${ }^{18}$ Many studies investigating TLR signaling mechanisms at the ocular surface have employed monolayer cell cultures that are nonstratified and undifferentiated, where the expression of MAMs, especially that of MUC16, does not occur. ${ }^{30}$ Thus, the effect of MAMs in modulating TLR signaling may have been underappreciated.

As a model system in this study, cultured human corneallimbal epithelial (HCLE) cells that (i) have been stratified and allowed to differentiate for optimal MAM expression and (ii) mimic several characteristics of the native corneal epithelium upon stratification were used. ${ }^{30}$ As a first step, the spectrum of TLRs expressed by differentiated HCLE cells was determined. By performing reverse transcriptase-PCR (RT-PCR) analyses, nontransfected HCLE (HCLE-NT) cells were found to express TLRs 2, 5, 6, and 10 (Figure 1). A very faint expression of TLR4 was also observed. Importantly, an identical TLR expression profile was observed when complementary DNA (cDNA) derived from fresh human corneal epithelium, removed at the time of surgery, was used as template (Figure 1). HCLEscrMUC1 and HCLE-scrMUC16 (scramble-transfected HCLE cells, controls), and HCLE-shMUC1 and HCLE-shMUC16 (MUC1 and MUC16 knockdown HCLE cells) were also found to mimic the TLR expression profile of HCLE-NT cells (data not shown). As TLR2 and TLR5 transcripts were found to be highly expressed in HCLE cells (Figure 1), agonists specific to these receptors were chosen for subsequent experiments. The reason for not choosing agonists to TLR6 and TLR10 was

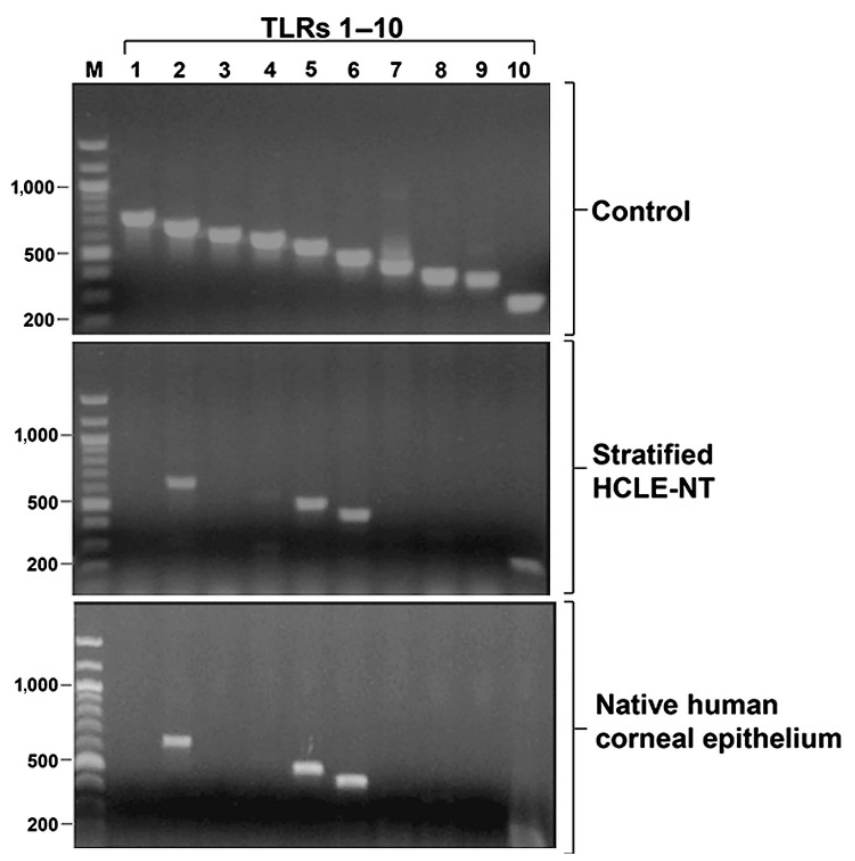

Figure 1 Expression of Toll-like receptors (TLRs) by differentiated corneal epithelial cells. Reverse transcriptase-PCR (RT-PCR) analysis indicates that differentiated nontransfected HCLE (HCLE-NT) cells express TLRs 2, 5, 6, and 10. An identical expression profile was observed in native corneal epithelial cells. In the figure, the panel labeled "Control" included complementary DNA (cDNA) from a human monocytic cell line as template in the reactions. DNA molecular weight standards in kilobase pairs are indicated on the left of the gel. 
because these receptors are functional only upon association with TLR2, and neither ligand specificity nor function of TLR10 has been defined. ${ }^{31,32}$

\section{TLR2 and TLR5 are expressed by apical and subapical corneal epithelial cells}

Three independent studies have reported that TLR5 is expressed by basal and wing cells of the corneal epithelium, ${ }^{15,28,33}$ whereas another study by Li et $a l .{ }^{34}$ demonstrated that the distribution of this receptor is relatively uniform across the entire human corneal epithelium. To clarify, the distribution of TLR5 and of TLR2 in the native human corneal epithelium and in differentiated HCLE-NT cells was examined. By immunohistochemical staining of cryostat sections of freshly isolated, noncultured, human corneal epithelial sheets removed during refractive surgery, both apical and subapical binding of TLR2and TLR5- specific antibodies was observed (Figure 2a,b). Using a second in vitro method, where surface proteins of HCLE-NT cells were biotin labeled, recovered, and analyzed by immunoblotting, TLR2 and TLR5 were detected on the apical surface of stratified HCLE cell cultures (Figure 2c,d).

\section{Expression of IL-6, IL-8, and TNF- $\alpha$ is elevated in HCLE-shMUC1 and HCLE-shMUC16 cells following stimulation by TLR2 and TLR5 agonists}

To test the hypothesis that the MAMs MUC1 and MUC16 suppress TLR-mediated innate immune responses, the responsiveness of differentiated MUC1- and MUC16-knockdown HCLE cells to agonists specific to TLR2 and TLR5 was investigated. As outcomes of TLR2 and TLR5 signaling, the levels of the proinflammatory cytokines IL-6, IL- 8 , and TNF- $\alpha$ in the different cell lines was measured by quantitative RT-PCR (qRT-PCR). Following exposure of HCLE-NT, HCLE-scrMUC1, HCLE-shMUC1, HCLE-scrMUC16, and HCLE-shMUC16 to the agonists for $4 \mathrm{~h}$, the message levels of IL-6, IL-8, and TNF- $\alpha$ were several fold higher (ranging from twofold to sixfold) in HCLE-shMUC1 and HCLE-shMUC16 cells in comparison with the controls (Figure 3a,b). Under conditions where HCLE cells were not exposed to any agonist, the message levels of IL- 6 , IL- 8 , and TNF- $\alpha$ remained comparable among the different cell lines (Supplementary Figure S1 online), indicating that these cytokines are not constitutively overexpressed in the knockdown cells. Also, the levels of IL-6, IL-8, and TNF- $\alpha$ secreted into the culture medium by the different cells were measured following a 12-h agonist exposure. HCLE-shMUC1 and HCLE-shMUC16 cells were found to secrete significantly higher levels of IL-6, IL-8, and TNF- $\alpha$ in comparison with control cells (Figure 3c,d).

\section{TLR2 and TLR5 mRNA/protein expression and surface distribution is not increased in MUC1- and MUC16- knockdown cells}

The elevated expression of IL-6, IL-8, and TNF- $\alpha$ observed in HCLE-shMUC1 and HCLE-shMUC16 cells following agonist exposure could be a result of increased TLR2 and TLR5 expression in these cells. To investigate if this was the case, mRNA and protein levels of TLR2 and TLR5 were compared in the different cell lines. The qRT-PCR and immunoblot analyses indicated that the expression of TLR2 and TLR5 is not increased in either HCLE-shMUC1 or HCLE-shMUC16 cells in comparison with HCLE-NT, HCLE-scrMUC1, and HCLEscrMUC16 cells (Figure 4a,b). Although TLR2 and TLR5 were found to be uniformly expressed by the different cell lines, perhaps the surface distribution of these receptors may be increased in HCLE-shMUC1 and HCLE-shMUC16 cells, and this could also explain the elevated expression of IL-6, IL-8, and TNF- $\alpha$ in these cells following agonist exposure. To test this possibility, cell surface biotinylation experiments were
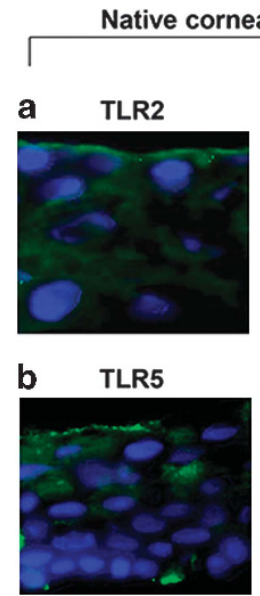

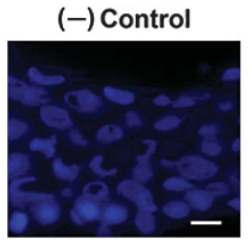

\section{(-) Control}

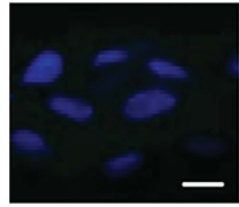

$(-)$ Control
Stratified HCLE-NT cells

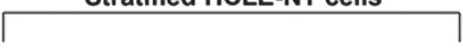

c

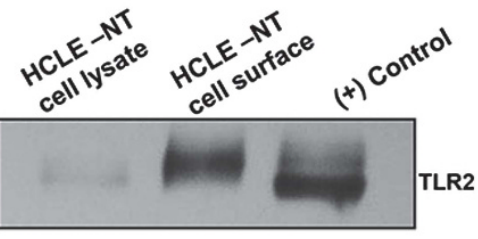

d

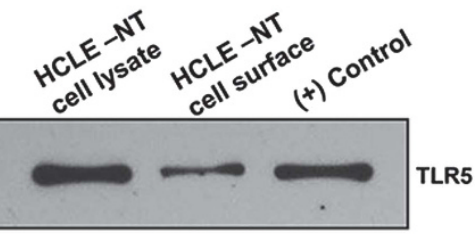

Figure 2 Distribution of Toll-like receptors 2 and 5 (TLR2 and TLR5) across the corneal epithelium. (a,b) Immunofluorescence microscopy on crosssection of freshly obtained, noncultured, human corneal epithelium using goat polyclonal anti-TLR2 antibody and mouse monoclonal anti-TLR5 antibody revealed that TLR2 and TLR5 are expressed by apical and subapical cells of the stratified corneal epithelium. In the control condition, incubation was performed using the fluorescein isothiocyanate (FITC)-labeled secondary antibody in the absence of primary antibody. Nuclei are localized using 4',6diamidino-2-phenylindole (DAPI). Scale bars $=10 \mu \mathrm{m}$. (c,d) Western blots confirming that TLR2 and TLR5 are expressed on the surface of stratified nontransfected HCLE (HCLE-NT) cells as determined by cell surface biotinylation (middle lanes labeled HCLE-NT cell surface). The lanes labeled HCLENT cell lysate and $(+)$ Control contained $5 \mu \mathrm{g}$ total protein. The $(+)$ Control used was a lysate derived from undifferentiated THP-1 cells. 
a

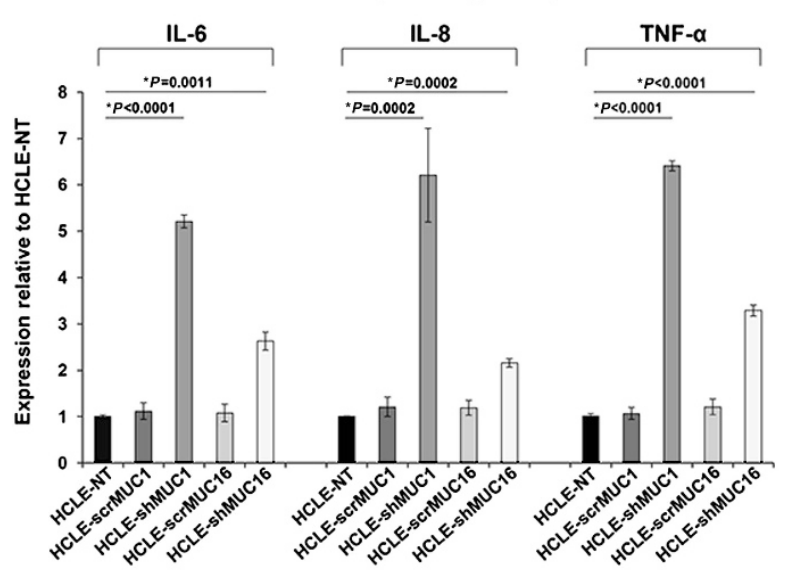

c

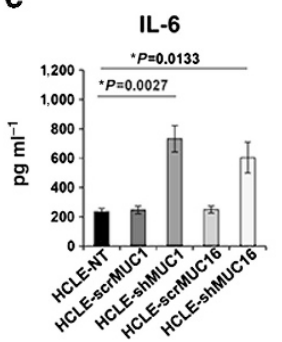

HKLM (TLR2 agonist)

b

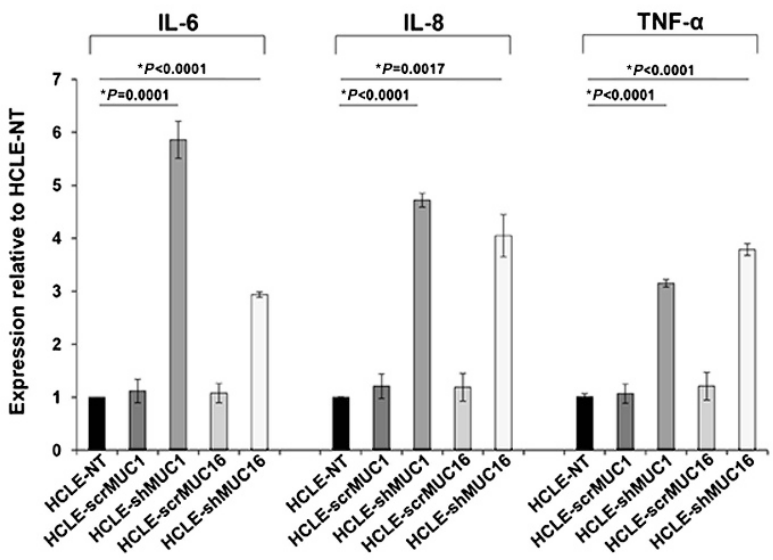

d

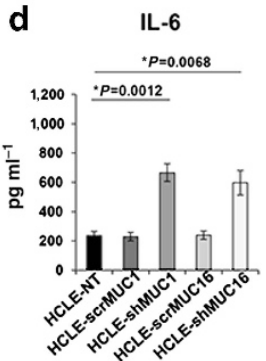

Flagellin (TLR5 agonist)

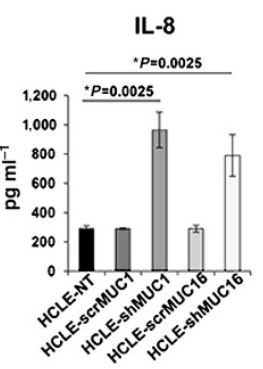

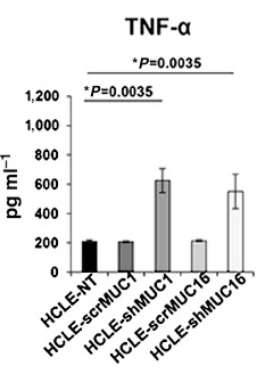

Figure 3 Expression of proinflammatory cytokines in nontransfected HCLE (HCLE-NT), HCLE-scrMUC1, HCLE-shMUC1, HCLE-scrMUC16, and HCLE-shMUC16 cells following exposure to Toll-like receptor 2 and 5 (TLR2 and TLR5) agonists. (a,b) Quantitative reverse transcriptase-PCR (qRTPCR) analysis indicates that the message levels of the proinflammatory cytokines interleukin (IL)-6, IL-8, and tumor necrosis factor- $\alpha$ (TNF- $\alpha$ ) are increased in HCLE-shMUC1 and HCLE-shMUC16 cells in comparison with HCLE-NT, HCLE-scrMUC1, and HCLE-scrMUC16 cells following exposure of these cells to the TLR2 and TLR5 agonists, HKLM (a heat-killed preparation of Listeria monocytogenes) and flagellin, respectively. Glyceraldehyde 3phosphate dehydrogenase (GAPDH) was used as endogenous control in the qRT-PCRs. (c,d) Results from Luminex assays indicate that HCLEshMUC1 and HCLE-shMUC16 cells secrete increased levels of IL-6, IL-8, and TNF- $\alpha$ following a 12-h exposure of the cells to HKLM and flagellin. Data shown are from experiments performed in biological triplicates. One-way analysis of variance (ANOVA) was done to determine overall significance between groups. For internal comparisons, unpaired $t$-tests with Bonferroni correction were performed. ${ }^{*} P<0.025$ was considered significant.

performed. Results showed that the surface distribution of TLRs 2 and 5 remained unaltered between the different cell lines (Figure 4c), indicating that the increased expression of IL-6, IL-8, and TNF- $\alpha$ observed in the knockdown cells is more likely due to lack of MUC1 or MUC16.

\section{IL-6 and TNF- $\alpha$ transcripts are elevated in the corneal epithelium of Muc1 ${ }^{-/-}$mice following exposure to the TLR2 and TLR5 agonists}

To extend the in vitro observations, ex vivo experiments using eyes derived from $\mathrm{Muc1}^{-/}$mice were performed. If MUC1 suppresses TLR signaling at the ocular surface, as seen in HCLE-shMUC1 cells, then the corneal epithelium of Mucl $^{-/-}$mice should exhibit a heightened TLR response following agonist challenge. Indeed, exposure of the enucleated eyes derived from $\mathrm{Muc1}^{-1-}$ mice to a cocktail of the TLR2 and TLR5 agonists for $4 \mathrm{~h}$ resulted in increased message levels of IL- 6 and TNF- $\alpha$ in the corneal epithelium as compared with the WT condition (Figure 5).

To determine whether Mucl $^{-1-}$ mice have an ocular surface phenotype and/or exhibit signs of ocular inflammation, fluorescein staining of the ocular surface to assess epithelial defects and tear volume measurements was performed. No significant differences between WT and $\mathrm{Mucl}^{-/-}$mice were noted (Supplementary Figure S3A, B). Furthermore, expression of Sprr2h and Tgm 1 (markers of epithelial stress and keratinization, respectively) ${ }^{35}$ in the corneal epithelium did not differ significantly between WT and $\mathrm{Mucl}^{-/-}$mice (Supplementary Figure S4). Message levels of Muc4, another MAM expressed by the corneal epithelium of mice, ${ }^{36}$ were also found to be comparable (Supplementary Figure S4), suggesting that Muc4 is not upregulated in response to loss of Mucl in the knockout mice. TNF- $\alpha$ (indicative of inflammation) message levels were found to be significantly lower in the corneal epithelium of $\mathrm{Mucl}^{-/-}$mice in comparison with WT mice (Supplementary Figure S4). Immunohistochemical analyses for determining the presence of inflammatory cells in the ocular surface tissue using the pan-leukocyte marker CD45 revealed that the number of CD45-positive cells per $1 \mathrm{~mm}$ linear length of basal lamina in the corneal epithelium (WT: $0.1 \pm 0.1, \mathrm{Mucl}^{-/-}: 0$ ) and corneal stroma (WT: $0.9 \pm 0.3, \mathrm{Mucl}^{-/-}$: $0.4 \pm 0.1$ ) were similar between WT and Muc1 ${ }^{-1-}$ mice. Taken together, these data suggest that $\mathrm{C} 57 \mathrm{BL} / 6 \mathrm{Mucl}^{-/-}$mice do not display an 


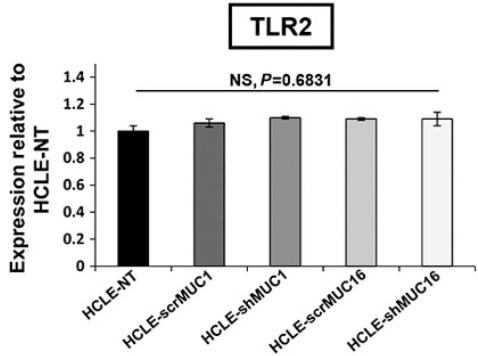

b
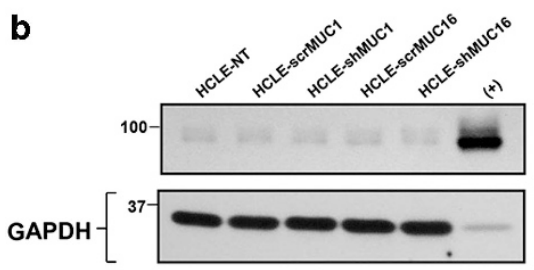

c

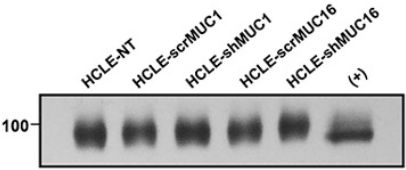

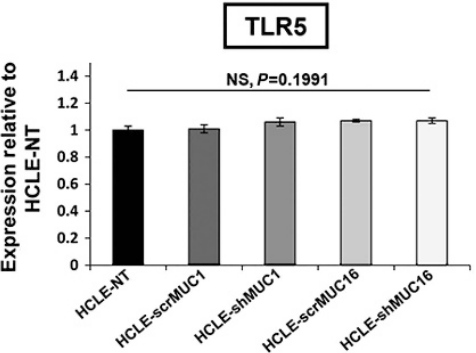
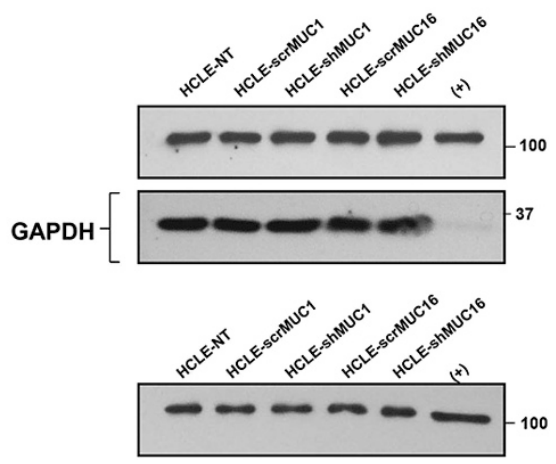

Figure 4 The mRNA/protein expression and cell surface distribution of Toll-like receptors 2 and 5 (TLR2 and TLR5) in HCLE-shMUC1 and HCLEshMUC16 cells. (a) Quantitative reverse transcriptase-PCR (qRT-PCR) and (b) immunoblot analyses demonstrate that the expression of TLR2 and TLR5 in HCLE-shMUC1 and HCLE-shMUC16 cells are not significantly different from HCLE-NT, HCLE-scrMUC1, and HCLE-scrMUC16 cells. Data shown in a are from experiments performed in biological triplicates. One-way analysis of variance (ANOVA) was done to determine overall significance between groups. For internal comparisons, unpaired $t$-tests with Bonferroni correction were performed. $P>0.025$ was considered not significant (NS). In b, whole-cell extracts $(5 \mu \mathrm{g})$ corresponding to HCLE-NT, HCLE-shMUC1, HCLE-scrMUC1, HCLE-scrMUC16, and HCLE-shMUC16 cells were resolved by $12 \%$ SDS-polyacrylamide gel electrophoresis, followed by electroblotting and immunoblotting using TLR2- and TLR5-specific antibodies.

Glyceraldehyde 3-phosphate dehydrogenase (GAPDH) served as the loading control. (c) Immunoblot showing that the apical distribution of TLR2 and TLR5 does not change between HCLE-NT, HCLE-shMUC1, HCLE-scrMUC1, HCLE-scrMUC16, and HCLE-shMUC16 cells as determined by cell surface biotinylation experiments. The lanes labeled $(+)$ in $\mathbf{b}$ and $\mathbf{c}$ correspond to a lysate derived from undifferentiated THP-1 cells $(5 \mu \mathrm{g})$.

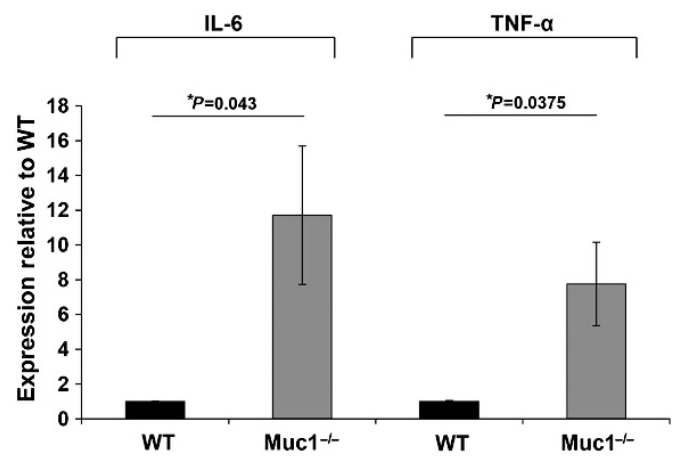

Figure 5 Expression of interleukin-6 (IL-6) and tumor necrosis factor- $\alpha$ $(\mathrm{TNF}-\alpha)$ in the corneal epithelium of Muc1 ${ }^{-1}{ }^{-}$mice following exposure to a cocktail of Toll-like receptor 2 and 5 (TLR2 and TLR5) agonists. Quantitative reverse transcriptase-PCR (qRT-PCR) analysis indicates that IL-6 and TNF- $\alpha$ message levels in the corneal epithelia of Muc1 ${ }^{-1-}$ mice (knockout (KO), gray, $n=6$ ) are elevated in comparison with WT mice (black, $n=3$ ) following exposure of enucleated eyes to a cocktail of the TLR2 and TLR5 agonists, HKLM (a heat-killed preparation of Listeria monocytogenes) and flagellin, respectively. Mouse glyceraldehyde 3-phosphate dehydrogenase (GAPDH) was used as endogenous control in the qRT-PCRs. Unpaired $t$-tests with Welch correction were performed to determine significance. ${ }^{*} P<0.05$ was considered significant.

ocular surface phenotype or signs of ocular inflammation, a finding that is consistent with the study published by Danjo et al. ${ }^{36}$

\section{DISCUSSION}

This study demonstrates that the MAMs MUC16 and MUC1 each modulate inflammatory responses at the corneal epithelium by limiting TLR2- and TLR5-induced expression of the proinflammatory cytokines IL-6, IL-8, and TNF- $\alpha$ (Figure 6). Although the barrier properties of MUC16 have been previously reported, ${ }^{8,9}$ this study is the first to assign an immunomodulatory role for MUC16. A similar function for MUC1 at the corneal epithelium is also described.

The immunomodulatory function of MUC16 may be explained, in part, by the barrier function of the glycoprotein. Studies have shown that at the corneal epithelium (i) MUC16 is antiadhesive and prevents colonization of the bacterium Staphylococcus aureus, ${ }^{8,9}$ (ii) glycosylation of the MAM and its binding to galectin-3 is crucial for the maintenance of mucosal barrier function, ${ }^{37}$ and (iii) knockdown of MUC16 in corneal epithelial cells results in reduced transepithelial resistance and disruption of tight junctions. ${ }^{9}$ Alternatively, immunosuppression may also be mediated by the CT of MUC16 through direct association with TLRs and/or inhibition of downstream signaling pathways. The CT of MUC16, which contains potential serine/threonine/tyrosine phosphorylation sites, was recently found to interact with the Src family of kinases (c-Src and c-Yes), ${ }^{38}$ which are proteins involved in TLR signaling. ${ }^{39}$ Perhaps such interactions limit the participation of Src in TLR 

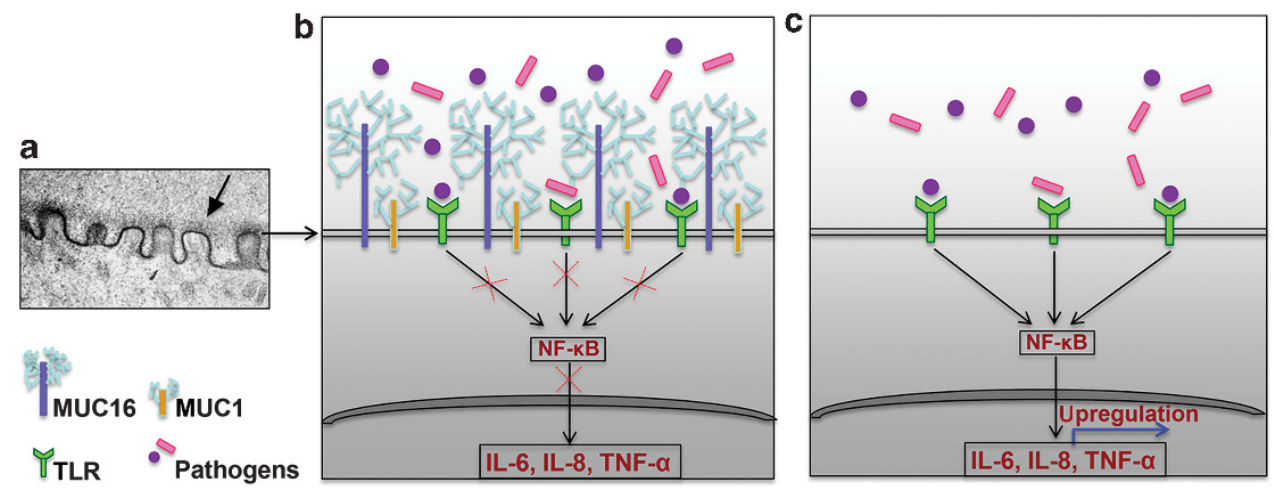

Figure 6 Schematic of MUC1- and MUC16-mediated suppression of Toll-like receptor (TLR) signaling at the corneal epithelium. (a) Membraneassociated mucins (MAMs) form a glycocalyx on the apical membrane of the corneal epithelium. The micrograph shows an electron-dense MAM glycocalyx (solid arrow) emanating from surface microplicae. ${ }^{58}$ (b) Although the precise mechanisms by which MUC1 and MUC16 mediate suppression of TLR signaling at the corneal epithelium remain to be determined, both MAMs limit unnecessary TLR activation and expression of the proinflammatory cytokines interleukin (IL)-6, IL-8, and tumor necrosis factor- $\alpha$ (TNF- $\alpha$ ). (c) Lack of MAMs results in increased TLR-induced expression of IL-6, IL-8, and TNF- $\alpha$.

signaling. Another possibility is that MUC16-CT, upon phosphorylation, may directly modulate NF- $\mathrm{\kappa B}$ activity and expression of proinflammatory cytokines.

The finding that MUC1 suppresses TLR responses at the corneal epithelium is noteworthy as the protein's function at the ocular surface has remained elusive thus far. Previously, Kardon et al. ${ }^{40}$ reported that C57BL/6 $\times$ SVJ129 $\mathrm{Mucl}^{-1-}$ mice developed spontaneous conjunctivitis and blepharitis; however, a subsequent study by Danjo et al. ${ }^{36}$ showed no increased susceptibility of $\mathrm{C} 57 \mathrm{BL} / 6 \mathrm{Muc1}^{-1-}$ mice to infection by $P$ aeruginosa. This discrepancy may be because of strain variation, and it is possible that $\mathrm{C} 57 \mathrm{BL} / 6 \mathrm{Muc1}{ }^{-1-}$ mice are more resistant to infections, as noted by Kardon et al. ${ }^{40}$ In this study, at steady state, $\mathrm{C} 57 \mathrm{BL} / 6 \mathrm{Mucl}^{-1-}$ mice did not exhibit an ocular surface phenotype or signs of inflammation (Supplementary Data), consistent with the findings of Danjo et al. ${ }^{36}$ Although MUC1 is a critical component of the mucosal barrier in the gastric epithelium, ${ }^{41,42}$ loss of MUC1 in corneal epithelial cells correlates with increased barrier function. ${ }^{9}$ Thus, rather than serving as a barrier at the corneal epithelium, MUC1 may exert its immunosuppressive function via other mechanisms. Kato et al. ${ }^{26}$ demonstrated that the epidermal growth factor (EGF) receptor kinase phosphorylates a tyrosine residue in the CT of MUC1 that increases its association with TLR5 and blocks MyD88 recruitment to the receptor. This association is found to inhibit downstream signal transduction. ${ }^{26}$ More recently, Sheng et al. ${ }^{21}$ reported that MUC1 inhibits inflammatory responses of gastric epithelial cells to TNF- $\alpha$ and to TLR/NOD1 ligands by suppressing $\mathrm{NF}-\kappa \mathrm{B}$ activity. Based on these reports, the mechanism of MUC1-mediated suppression of TLR responses at the corneal epithelium needs to be further evaluated.

The in vitro data that loss of either MUC1 or MUC16 leads to increased TLR responses is a conundrum, as the knockdown cells express normal levels of one of these MAMs. ${ }^{9}$ However, this is not entirely surprising as, in primary human bronchial epithelial cells, where both MUC1 and MUC16 are expressed, ${ }^{43}$ knockdown of MUC1 alone results in enhanced flagellin- induced IL- 8 synthesis. ${ }^{24}$ Perhaps, when expressed together, the CTs of both MUC1 and MUC16 interact with a common protein that is involved in TLR signaling, and when expression of either MAM is reduced/lost, dissociation of this protein triggers an elevated TLR response. One such protein may be c-Src as it has been shown to bind to both MUC1 and MUC16. ${ }^{38,44}$ As MUC16 does not contain an EGF-like domain in its transmembrane domain, ${ }^{45}$ the possibility of EGF receptor being a common interaction partner for both MUC1 and MUC16 can be ruled out.

Previous observations that TLRs 2 and 5 are expressed within the internal layers of the corneal epithelium led to the hypothesis that activation of these receptors occurs only upon compromise of the epithelial barrier. ${ }^{15,16,28,33}$ In this study, TLRs 2 and 5 were found to be expressed by apical and subapical cells of the corneal epithelium. Based on this observation and the finding that reduced expression of MAMs on the apical surface of corneal epithelial cells leads to increased TLR-induced expression of proinflammatory cytokines, it is proposed that both MUC1 and MUC16 play a role in keeping TLR responses in check. The activation state of NF- $\kappa B$ was not assayed in this study. As all TLR signaling pathways, MyD88 or TRIF dependent, converge on NF- $\kappa B$ and ultimately lead to the synthesis of several downstream cytokines including IL-6, IL-8, and TNF- $\alpha$, the expression of these cytokines was assayed as an outcome of TLR signaling.

In conclusion, findings reported herein suggest a role for both MUC1 and MUC16 in preventing unnecessary TLR activation and signaling, and maintenance of immune homeostasis at the ocular surface. Future studies will investigate the molecular bases of immunosuppression by MUC1 and MUC16 at the ocular surface.

\section{METHODS}

Cell lines and culture methods. A telomerase-transformed HCLE cell line for which mucin gene expression has been well characterized was used as an in vitro model. ${ }^{8,30,46}$ The HCLE-NT cells were cultured and grown to confluence in keratinocyte serum-free medium (K-SFM) 
(Invitrogen, Carlsbad, CA) containing $25 \mu \mathrm{g} \mathrm{ml}^{-1}$ bovine pituitary extract and $0.2 \mathrm{ng} \mathrm{ml}^{-1}$ EGF. Cells were then switched to Dulbecco's modified Eagle's medium/Nutrient Mixture F-12 (Cellgro, Manassas, VA) supplemented with $10 \%$ calf serum and $10 \mathrm{ng} \mathrm{ml}^{-1}$ EGF for 7 days to promote differentiation and optimal MAM production. ${ }^{30}$ Methods for generating the HCLE-scrMUC1, HCLE-shMUC1, HCLE-scrMUC16, and HCLE-shMUC16 cells have been described previously. ${ }^{8,9}$ The HCLE-scrMUC1/HCLE-shMUC1 and HCLEscrMUC16/HCLE-shMUC16 cells were grown and maintained in medium containing blasticidin $\left(5 \mu \mathrm{g} \mathrm{ml}^{-1}\right)$ and puromycin $\left(2.5 \mu \mathrm{g} \mathrm{ml}^{-1}\right)$, respectively.

Monocytic THP- 1 cells were grown to a density of $1 \times 10^{6} \mathrm{cells} \mathrm{ml}^{-1}$ in RPMI-1640 medium supplemented with $10 \%$ fetal bovine serum, penicillin, and streptomycin, and $10 \mathrm{~mm}$ L-glutamine. Differentiation of THP-1 cells was achieved by treating cells with $100 \mathrm{~nm}$ phorbol 12-myristate 13-acetate for 3 days.

Mouse models. Male WT and Muc1 ${ }^{-1-}$ mice, 8 to 12 weeks old, both on a C57BL/6 genetic background, were used in in vivo and ex vivo studies. These mice were obtained from the laboratory of Dr Sandra Gendler (Mayo Clinic, Scottsdale, AZ). Details regarding generation of the $\mathrm{Mucl}^{-1-}$ mice have been described previously. ${ }^{49}$ All animal protocols were approved by the Schepens Institutional Animal Care and Use Committee. Muc16 ${ }^{-1-}$ mice were not used in this study, as mice do not express Muc16 in the corneal epithelium. ${ }^{48}$

TLR2 and TLR5 agonist exposure in vitro and ex vivo. Agonists to TLR2 (a heat-killed preparation of Listeria monocytogenes (HKLM)) and TLR5 (flagellin from Salmonella typhimurium) were purchased from InvivoGen (San Diego, CA). These agonists were used at working concentrations recommended by the manufacturer (HKLM $10^{8}$ cells per $\mathrm{ml}$, flagellin $250 \mathrm{ng} \mathrm{ml}^{-1}$ ). At $24 \mathrm{~h}$ before agonist exposure, stratified HCLE-NT, HCLE-scrMUC1, HCLE-shMUC1, HCLEscrMUC16, and HCLE-shMUC16 cells were switched to antibioticand EGF-free K-SFM. Cells were then exposed to the agonists that were prediluted in antibiotic- and EGF-free K-SFM for either $4 \mathrm{~h}$ (RNA analyses) or $12 \mathrm{~h}$ (protein analysis). In ex vivo experiments, eyes of WT and $\mathrm{Mucl}^{-1-}$ mice were enucleated and independently incubated in antibiotic- and EGF-free K-SFM containing a cocktail of the TLR2 and TLR5 agonists at working concentrations described above. Following a 4-h exposure to the agonists, the corneal epithelium was carefully debrided using a blade attached to a micro blade holder. The debrided epithelium from four eyes of each strain was pooled to constitute one sample (total $n=3$ for WT and $n=6$ for Muc1 ${ }^{-1-}$, each $n=4$ eyes). Pooled samples were collected in RNA lysis buffer (Qiagen, Valencia, $\mathrm{CA}$ ) and stored at $-80^{\circ} \mathrm{C}$ until further use.

RNA extraction and qRT-PCR analyses. Total RNA from stratified HCLE-NT, HCLE-scrMUC1, HCLE-shMUC1, HCLE-scrMUC16, HCLE-shMUC16, and native corneal epithelial cells was extracted using Trizol reagent (Life Technologies, Carlsbad, CA). Using the iScript cDNA synthesis kit (Bio-Rad, Waltham, MA), $2 \mu \mathrm{g}$ of total RNA was reverse-transcribed following the manufacturer's guidelines. For amplifying TLRs, cDNA corresponding to the different HCLE cell lines and native corneal epithelial cells was used as template in PCRs along with TLR-specific primers that were purchased from InvivoGen (Human TLR RT-primers). The positive control used in PCRs was double-stranded DNA from monocytic THP-1 cells (InvivoGen). Amplified products were resolved on $1.2 \%$ agarose gels containing ethidium bromide. The qRT-PCR to measure transcript levels of IL-6, IL-8, TNF- $\alpha$, TLR2, and TLR5 was performed on an Eppendorf Mastercycler ep gradient S platform using TaqMan chemistry and prevalidated primers from Life Technologies (IL-6 (Hs00985639_m1), IL-8 (Hs00174103_m1), TNF- $\alpha$ (Hs01113624_g1), TLR2 (Hs01872448_s1), TLR5 (Hs01920773_s1), and GAPDH (4333764F)). Data were normalized to the endogenous control, glyceraldehyde 3phosphate dehydrogenase (GAPDH), and expressed relative to HCLENT cells.
RNA from the debrided corneal epithelium of WT and $\mathrm{Mucl}^{-1-}$ mice was isolated using the RNeasy Micro kit (Qiagen). The cDNA synthesis and qRT-PCR analyses to measure transcript levels of IL-6 and TNF- $\alpha$ were performed as described above. Prevalidated primers purchased from Life Technologies were: IL-6 (Mm00446190_ m1), TNF- $\alpha$ (Mm00443258_m1), Sprr2h (Mm00488435_s1), Tgm1 (Mm00498375_m1), Muc1 (Mm00449604_m1), Muc4 (Mm00466886_m1), and GAPDH (Mm99999915_g1). Data were normalized to the endogenous control, GAPDH, and expressed relative to WT mice.

Luminex assays for measuring secreted cytokines. IL-6, IL-8, and TNF- $\alpha$ secreted into the culture medium by HCLE-NT, HCLEscrMUC1, HCLE-shMUC1, HCLE-scrMUC16, and HCLE-shMUC16 cells were detected and quantified using the Luminex Performance Assay (R\&D Systems, Minneapolis, MN) following the manufacturer's guidelines. Cells were exposed to the TLR2 and TLR5 agonists for $12 \mathrm{~h}$. For quantifying IL-8, 1/20th volume of the cell culture supernatants was used in the assay and for IL- 6 and TNF- $\alpha$ measurements, half volume of the culture supernatants was concentrated using a $10-\mathrm{kDa}$ cutoff concentrator (Millipore, Cork, Ireland). The concentration step was performed to ensure that IL- 6 and TNF- $\alpha$ levels were within range of the standard curve. Samples were analyzed using a Bio-Rad Bio-Plex analyzer powered by Luminex 100 xMAP technology (Luminex, Austin, TX). Resulting fluorescent intensities were used to calculate the concentrations of IL-6, IL- 8 , and TNF- $\alpha$ from a standard curve.

Immunohistochemistry of TLR2 and TLR5. Fresh, noncultured, human corneal epithelial sheets, removed by epikeratome for corrective refractive surgery and frozen in optimal cutting temperature medium within $1 \mathrm{~h}$ after surgery, were processed for immunohistochemistry. For immunolocalization of TLR2 and TLR5, $6 \mu \mathrm{m}$ cryostat sections were incubated with either rabbit polyclonal anti-TLR2 IgG $(\mathrm{H} 175)^{49,50}$ primary antibody (1:50 dilution; Santa Cruz Biotechnology, Paso Robles, CA) overnight at $4{ }^{\circ} \mathrm{C}$ in a moist chamber or mouse monoclonal anti-TLR5 IgG (19D759.2) ${ }^{33,51}$ primary antibody (1:100 dilution; Abcam, Cambridge, MA) for $1 \mathrm{~h}$ at room temperature, followed by either fluorescein isothiocyanatelabeled donkey anti-rabbit or anti-mouse IgG as the secondary antibody (1:100 dilution; Jackson ImmunoResearch, West Grove, PA) for $1 \mathrm{~h}$ at room temperature. After final washing with phosphatebuffered saline, slides were mounted in Vectashield medium containing 4',6-diamidino-2-phenylindole. Stained sections were viewed under a Zeiss (Thornwood, NY) Photoscope III fluorescence microscope. Specificities of the polyclonal anti-TLR2 and monoclonal anti-TLR5 antibodies were determined by immunoblotting using clarified cell extracts derived from undifferentiated and differentiated THP-1 cells, ${ }^{52,53}$ human umbilical vein endothelial cells, ${ }^{54,55}$ and HCLE-NT cells (Supplementary Figure S2).

Cell surface biotinylation and immunoblotting. Surface biotinylation of HCLE-NT, HCLE-scrMUC1, HCLE-shMUC1, HCLE-scrMUC16, and HCLE-shMUC16 cells was performed using the Pierce Pinpoint Cell Surface Protein Isolation Kit (Thermo Scientific, Rockford, IL). ${ }^{46,56,57}$ Biotinylated proteins were separated on $12 \%$ SDS-polyacrylamide gels, electroblotted onto nitrocellulose membranes, and analyzed by immunoblotting. Primary antibodies used were rabbit monoclonal anti-TLR2 IgG (EPNCIR133, 1:2,000 dilution; Abcam), rabbit monoclonal anti-TLR5 IgG (EPR10373, 1:2,000 dilution; Abcam), and rabbit polyclonal anti-GAPDH IgG (FL-375, 1:20,000 dilution; Santa Cruz Biotechnology), whereas the secondary antibody used was horseradish peroxidase-conjugated goat anti-rabbit IgG (Santa Cruz Biotechnology). Blots were developed using the SuperSignal West Femto Maximum Sensitivity Substrate (Thermo Scientific). Clarified lysates obtained from undifferentiated THP-1 cells were used as a positive control for detecting TLR 2 and TLR 5 by immunoblotting. 
Statistical analyses. For experiments with cell lines, statistical analyses were performed initially using the one-way analysis of variance test to determine overall significance. For internal comparisons, unpaired $t$ tests were performed with the Bonferroni correction. When applying the Bonferroni correction, $P<0.05 / 2$ or 0.025 (because of two comparisons) was used as the cutoff for significance. For experiments where WT and $\mathrm{Mucl}^{-1-}$ mice were being compared, unpaired $t$-tests with or without the Welch correction was used. The Welch correction was used when the difference between two s.d. values was significant. Statistical analyses were performed using the GraphPad (La Jolla, CA) Instat 3 program for Macintosh, version 3.1a. Individual $P$-values are indicated in the figures.

SUPPLEMENTARY MATERIAL is linked to the online version of the paper at http://www.nature.com/mi

\section{ACKNOWLEDGMENTS}

We thank Dr Gendler and Ms Madsen for providing the C57BL/6 WT and Muc $1^{-1-}$ mice, Dr Jurkunas for providing human corneal epithelial sheets, Ms Guo for help with statistics, and Dr D'Amore for providing the THP-1 cells and HUVEC extract. This work was supported by grant R01EY018850 (NEI/NIH) to I.K.G. and a Harvard Cornea Center of Excellence Fellowship to B.B.M.

\section{DISCLOSURE}

The authors declared no conflict of interest.

C 2015 Society for Mucosal Immunology

\section{REFERENCES}

1. Perez-Vilar, J. \& Hill, R.L. The structure and assembly of secreted mucins. J. Biol. Chem. 274, 31751-31754 (1999).

2. Kufe, D.W. Mucins in cancer: function, prognosis and therapy. Nat. Rev. Cancer 9, 874-885 (2009).

3. Hattrup, C.L. \& Gendler, S.J. Structure and function of the cell surface (tethered) mucins. Ann. Rev. Physiol. 70, 431-457 (2008).

4. Bafna, S., Kaur, S. \& Batra, S.K. Membrane-bound mucins: the mechanistic basis for alterations in the growth and survival of cancer cells. Oncogene 29, 2893-2904 (2010).

5. Senapati, S., Das, S. \& Batra, S.K. Mucin-interacting proteins: from function to therapeutics. Trends Biochem. Sci. 35, 236-245 (2010).

6. Govindarajan, B. \& Gipson, I.K. Membrane-tethered mucins have multiple functions on the ocular surface. Exp. Eye Res. 90, 655-663 (2010).

7. Gipson, I.K. Distribution of mucins at the ocular surface. Exp. Eye Res. 78 , 379-388 (2004).

8. Blalock, T.D. et al. Functions of MUC16 in corneal epithelial cells. Invest. Ophthalmol. Vis. Sci. 48, 4509-4518 (2007).

9. Gipson, I.K., Spurr-Michaud, S., Tisdale, A. \& Menon, B.B. Comparison of the transmembrane mucins MUC1 and MUC16 in epithelial barrier function. PLoS One 9, e100393 (2014).

10. Keogh, B. \& Parker, A.E. Toll-like receptors as targets for immune disorders. Trends Pharmacol. Sci. 32, 435-442 (2011).

11. Kumar, A., Zhang, J. \& Yu, F.-S.X. Toll-like receptor 2-mediated expression of $\beta$-defensin-2 in human corneal epithelial cells. Microbes Infect. 8, 380-389 (2006).

12. Kumar, A., Zhang, J. \& Yu, F.S. Toll-like receptor 3 agonist poly(l:C)-induced antiviral response in human corneal epithelial cells. Immunology 117 , $11-21$ (2006).

13. Huang, X., Du, W., McClellan, S.A., Barrett, R.P. \& Hazlett, L.D. TLR4 is required for host resistance in Pseudomonas aeruginosa keratitis. Invest. Ophthalmol. Vis. Sci. 47, 4910-4916 (2006).

14. Johnson, A.C. et al. Activation of toll-like receptor (TLR)2, TLR4, and TLR9 in the mammalian cornea induces MyD88-dependent corneal inflammation. Invest. Ophthalmol. Vis. Sci. 46, 589-595 (2005).

15. Redfern, R.L., Reins, R.Y. \& McDermott, A.M. Toll-like receptor activation modulates antimicrobial peptide expression by ocular surface cells. Exp. Eye Res. 92, 209-220 (2011).
16. Ueta, M. et al. Intracellularly expressed TLR2s and TLR4s contribution to an immunosilent environment at the ocular mucosal epithelium. J. Immunol. 173, 3337-3347 (2004).

17. Ueta, M., Hamuro, J., Kiyono, H. \& Kinoshita, S. Triggering of TLR3 by polyl:C in human corneal epithelial cells to induce inflammatory cytokines. Biochem. Biophys. Res. Commun. 331, 285-294 (2005).

18. Redfern, R.L. \& McDermott, A.M. Toll-like receptors in ocular surface disease. Exp. Eye Res. 90, 679-687 (2010).

19. Yu, F.-S.X. \& Hazlett, L.D. Toll-like receptors and the eye. Invest. Ophthalmol. Vis. Sci. 47, 1255-1263 (2006).

20. Ueno, K. et al. MUC1 mucin is a negative regulator of toll-like receptor signaling. Am. J. Respir. Cell Mol. Biol. 38, 263-268 (2008).

21. Sheng, Y.H. et al. MUC1 and MUC13 differentially regulate epithelial inflammation in response to inflammatory and infectious stimuli. Mucosal Immunol. 6, 557-568 (2013).

22. Kim, K.C. \& Lillehoj, E.P. MUC1 mucin: a peacemaker in the lung. Am. J. Respir. Cell Mol. Biol. 39, 644-647 (2008).

23. Guang, W., Ding, H., Czinn, S.J., Kim, K.C., Blanchard, T.G. \& Lillehoj, E.P. Muc1 cell surface mucin attenuates epithelial inflammation in response to a common mucosal pathogen. J. Biol. Chem. 285, 20547-20557 (2010).

24. Lu, W. et al. Enhanced pulmonary clearance of Pseudomonas aeruginosa by Muc1 knockout mice. J. Immunol. 176, 3890-3894 (2006).

25. Williams, M.A. et al. Deletion of the mucin-like molecule Muc1 enhances dendritic cell activation in response to toll-like receptor ligands. J. Innate Immun. 2, 123-143 (2010).

26. Kato, K. et al. Membrane-tethered MUC1 mucin is phosphorylated by epidermal growth factor receptor in airway epithelial cells and associates with TLR5 to inhibit recruitment of MyD88. J. Immunol. 188, 2014-2022 (2012)

27. Kato, K., Lillehoj, E.P. \& Kim, K.C. MUC1 regulates epithelial inflammation and apoptosis by Polyl:C through inhibition of Toll/IL-1 receptor-domaincontaining adapter-inducing IFN-beta (TRIF) recruitment to Toll-like receptor 3. Am. J. Respir. Cell Mol. Biol. 51, 446-454 (2014).

28. Zhang, J., Xu, K., Ambati, B. \& Yu, F.-S.X. Toll-like receptor 5-mediated corneal epithelial inflammatory responses to Pseudomonas aeruginosa flagellin. Invest. Ophthalmol. Vis. Sci. 44, 4247-4254 (2003).

29. Song, P.I. et al. The expression of functional LPS receptor proteins CD14 and toll-like receptor 4 in human corneal cells. I Invest. Ophthalmol. Vis. SCi 42, 2867-2877 (2001).

30. Gipson, I.K., Spurr-Michaud, S., Argueso, P., Tisdale, A., Ng, T.F. \& Russo, C.L. Mucin gene expression in immortalized human corneal-limbal and conjunctival epithelial cell lines. Invest. Ophthalmol. Vis. Sci. 44, 24962506 (2003).

31. Nakao, Y. et al. Surface-expressed TLR6 participates in the recognition of diacylated lipopeptide and peptidoglycan in human cells. J. Immunol. 174, 1566-1573 (2005).

32. Guan, Y. et al. Human TLRs 10 and 1 share common mechanisms of innate immune sensing but not signaling. J. Immunol. 184, 5094-5103 (2010).

33. Hozono, Y. et al. Human corneal epithelial cells respond to ocularpathogenic, but not to nonpathogenic-flagellin. Biochem. Biophys. Res. Commun. 347, 238-247 (2006)

34. Li, J., Shen, J. \& Beuerman, R.W. Expression of toll-like receptors in human limbal and conjunctival epithelial cells. Mol. Vis. 13, 813-822 (2007).

35. Marko, C.K., Menon, B.B., Chen, G., Whitsett, J.A., Clevers, H. \& Gipson, I.K. Spdef null mice lack conjunctival goblet cells and provide a model of dry eye. Am. J. Pathol. 183, 35-48 (2013).

36. Danjo, Y., Hazlett, L.D. \& Gipson, I.K. C57BL/6 mice lacking Muc1 show no ocular surface phenotype. Invest. Ophthalmol. Vis. Sci. 41, 4080-4084 (2000).

37. Argueso, P., Guzman-Aranguez, A., Mantelli, F., Cao, Z., Ricciuto, J. \& Panjwani, N. Association of cell surface mucins with galectin-3 contributes to the ocular surface epithelial barrier. J. Biol. Chem. 284, 23037-23045 (2009).

38. Akita, K., Tanaka, M., Tanida, S., Mori, Y., Toda, M. \& Nakada, H. CA125/ MUC16 interacts with Src family kinases, and over-expression of its C-terminal fragment in human epithelial cancer cells reduces cell-cell adhesion. Eur. J. Cell Biol. 92, 257-263 (2013).

39. Page, T.H., Smolinska, M., Gillespie, J., Urbaniak, A.M. \& Foxwell, B.M. Tyrosine kinases and inflammatory signalling. Curr. Mol. Med. 9, 69-85 (2009). 
40. Kardon, R. et al. Bacterial conjunctivitis in Muc1 null mice. Invest. Ophthalmol. Vis. Sci. 40, 1328-1335 (1999).

41. McAuley, J.L. et al. MUC1 cell surface mucin is a critical element of the mucosal barrier to infection. J. Clin. Invest. 117, 2313-2324 (2007).

42. Lindén, S.K. et al. MUC1 limits Helicobacter pylori infection both by steric hindrance and by acting as a releasable decoy. PLoS Pathog. 5, e1000617 (2009).

43. Davies, J.R., Kirkham, S., Svitacheva, N., Thornton, D.J. \& Carlstedt, I. MUC16 is produced in tracheal surface epithelium and submucosal glands and is present in secretions from normal human airway and cultured bronchial epithelial cells. Int. J. Biochem. Cell Biol. 39, 1943-1954 (2007).

44. Li, Y., Kuwahara, H., Ren, J., Wen, G. \& Kufe, D. The c-Src tyrosine kinase regulates signaling of the human DF3/MUC1 carcinoma-associated antigen with GSK3 beta and beta-catenin. J. Biol. Chem. 276, 60616064 (2001).

45. Haridas, D., Ponnusamy, M.P., Chugh, S., Lakshmanan, I., Seshacharyulu, P. \& Batra, S.K. MUC16: molecular analysis and its functional implications in benign and malignant conditions. FASEB J 28, 4183-4199 (2014).

46. Govindarajan, B. et al. A metalloproteinase secreted by Streptococcus pneumoniae removes membrane mucin MUC16 from the epithelial glycocalyx barrier. PLoS One 7, e32418 (2012).

47. Spicer, A.P., Rowse, G.J., Lidner, T.K. \& Gendler, S.J. Delayed mammary tumor progression in Muc-1 null mice. J. Biol. Chem. 270, 30093-30101 (1995).

48. Wang, Y. et al. MUC16 expression during embryogenesis, in adult tissues, and ovarian cancer in the mouse. Differentiation 76, 1081-1092 (2008).

49. Ioannidis, I., Ye, F., McNally, B., Willette, M. \& Flano, E. Toll-like receptor expression and induction of type I and type III interferons in primary airway epithelial cells. J. Virol. 87, 3261-3270 (2013).
50. Martin, F.J. \& Prince, A.S. TLR2 regulates gap junction intercellular communication in airway cells. J. Immunol. 180, 4986-4993 (2008).

51. Yamada, K., Ueta, M., Sotozono, C., Yokoi, N., Inatomi, T. \& Kinoshita, S. Upregulation of Toll-like receptor 5 expression in the conjunctival epithelium of various human ocular surface diseases. Br. J. Ophthalmol. 98, 1116-1119 (2014).

52. Zarember, K.A. \& Godowski, P.J. Tissue expression of human Toll-like receptors and differential regulation of Toll-like receptor mRNAs in leukocytes in response to microbes, their products, and cytokines. J. Immunol. 168, 554-561 (2002).

53. Hijiya, N. et al. Possible involvement of toll-like receptor 4 in endothelial cell activation of larger vessels in response to lipopolysaccharide. Pathobiology 70, 18-25 (2002).

54. Talreja, J., Kabir, M.H., B Filla, M., Stechschulte, D.J. \& Dileepan, K.N. Histamine induces Toll-like receptor 2 and 4 expression in endothelial cells and enhances sensitivity to Gram-positive and Gram-negative bacterial cell wall components. Immunology 113, 224-233 (2004).

55. Maaser, C. et al. Human intestinal microvascular endothelial cells express Toll-like receptor 5: a binding partner for bacterial flagellin. J. Immunol. 172, 5056-5062 (2004).

56. Menon, B.B. \& Govindarajan, B. Identification of an atypical zinc metalloproteinase, ZmpC, from an epidemic conjunctivitis-causing strain of Streptococcus pneumoniae. Microb. Pathog. 56, 40-46 (2013).

57. Blalock, T.D., Spurr-Michaud, S.J., Tisdale, A.S. \& Gipson, I.K. Release of membrane-associated mucins from ocular surface epithelia. Invest. Ophthalmol. Vis. Sci. 49, 1864-1871 (2008).

58. Nichols, B.A., Chiappino, M.L. \& Dawson, C.R. Demonstration of the mucous layer of the tear film by electron microscopy. Invest. Ophthalmol. Vis. Sci. 26, 464-473 (1985). 